\title{
Retorno da Produção de ARroz IrRigado com Cultivares Convencionais após o Uso do Sistema Clearfield ${ }^{\circledR 1}$
}

\author{
Non-Tolerant Rice Production after Using the Clearfield ${ }^{\mathrm{IM}}$ Rice System
}

\author{
AVILA, L.A. ${ }^{2}$, TELÓ, G.M. ${ }^{3}$, FERREIRA, R.B. ${ }^{4}$, MARCHESAN, E. ${ }^{5}$, MACHADO, S.L.O. ${ }^{6}$, \\ ROSSATO, T.L. ${ }^{3}$, CEZIMBRA, D.M. ${ }^{4}$ e RIGÃO JR., G. ${ }^{4}$
}

\begin{abstract}
RESUMO - Os herbicidas imazethapyr + imazapic usados no sistema Clearfield ${ }^{\circledR}$ de produção de arroz irigado podem persistir no solo e afetar cultivares de arroz não tolerantes semeados em sucessão. O efeito sobre o arroz irrigado não tolerante pode variar de acordo com os genótipos e herbicidas utilizados no arroz cultivado em sucessão. Em vista disso, foi realizado um experimento com o objetivo de avaliar alternativas de cultivares e herbicidas para o retorno da produção de arroz não tolerante após o uso do sistema Clearfield. O experimento foi instalado em área de várzea sistematizada da Universidade Federal de Santa Maria (Santa Maria, RS) e conduzido no ano agrícola 2006/07. O delineamento experimental foi de blocos ao acaso, em esquema trifatorial, com três repetições. O fator A foi composto por dois sistemas de sucessão de cultivo de arroz irrigado nos primeiros três anos de cultivo (2003/04, 2004/05 e 2005/06): sistema de sucessão 1 - duas safras agrícolas com o uso do sistema Clearfield e aplicação de imazethapyr + imazapic (75 e 25 g i.a. ha-1) e uma safra com cultivo de arroz não tolerante, com aplicação de quinclorac; e sistema de sucessão 2 - três safras agrícolas com o uso do sistema Clearfield e aplicação do herbicida na mesma dose especificada anteriormente. $\mathrm{O}$ fator B foi representado pelos cultivares de arroz irrigado usados no quarto ano de cultivo (BR IRGA 409, IRGA 417, IRGA 422 CL e BRS 7 “TAIM"). O fator C foi representado por diferentes herbicidas aplicados na safra 2006/07: bispyribac-sodium, clomazone + propanil, cyhalofop-butyl, quinclorac e penoxsulam. O cultivo de arroz irrigado após o uso do sistema Clearfield $^{\circledR}$ por dois anos requer, pelo menos, uma safra agrícola sem a aplicação do herbicida (imazethapyr + imazapic), para evitar que o residual dos herbicidas afete a produtividade do arroz não tolerante. A redução de produtividade do arroz não tolerante causada pelo residual do herbicida (imazethapyr + imazapic) semeado após três anos de uso consecutivo do sistema Clearfield foi de 30\%. Não houve diferença entre os cultivares não tolerantes testados neste experimento quanto à suscetibilidade ao residual de imazethapyr + imazapic. Não houve aumento de fitotoxicidade nas plantas do arroz irrigado pela aplicação dos herbicidas no arroz irrigado não tolerante semeado na sucessão, porém ressalta-se a necessidade do uso de herbicidas que não sejam inibidores da enzima ALS para reduzir a pressão de seleção de plantas daninhas resistentes ou tolerantes a esses herbicidas.
\end{abstract}

Palavras-chave: fitotoxicidade, persistência no solo, produtividade de grãos.

\begin{abstract}
The herbicides imazethapyr and imazapic used in the Clearfield rice system can persist in the soil and affect non-tolerant rice cultivars sown in rotation. The effect on rice plants can vary according to the cultivar and herbicide used in rotation. Thus, a field experiment was conducted to evaluate cultivar and herbicide options when growing non-tolerant rice after using Clearfield rice system. The experiment was installed in the 2006/07 growing season, in a lowland area located in Santa Maria, Rio Grande do Sul, Southern Brazil. The experiment was in a randomized block design with three replications in a factorial scheme. Factor A included two succession systems of Clearfield rice and non-Clearfield rice in the first three growing seasons (2003/04, 2004/05 and 2005/06):
\end{abstract}

Recebido para publicação em 3.3.2009 e na forma revisada em 12.3.2010.

2 Engô-Agr ${ }^{\circ}$., Ph.D., Professor Adjunto do Dep. de Fitotecnia da Universidade Federal de Pelotas - UFPEL, Caixa Postal 354 , 96010-900 Pelotas-RS, Bolsista do CNPq, <laavilabr@gmail.com>; ${ }^{3}$ Engo-Agr ${ }^{\circ}$., Mestrando do Programa de Pós-graduação em Agronomia, Universidade Federal de Santa Maria - UFSM; ${ }^{4}$ Acadêmico de Agronomia, UFSM; ${ }^{5}$ Engo-Agr ${ }^{\circ}$., Dr., Professor Titular do Dep. de Fitotecnia, UFSM, Bolsista do CNPq; ${ }^{6}$ Engo$^{-}$Agro ${ }^{-}$, Dr., Professor Titular do Dep. de Defesa Fitossanitária da UFSM.

Planta Daninha, Viçosa-MG, v. 28, n. 1, p. 123-129, 2010 
Succession 1 - two agricultural growing seasons using the Clearfield system with application of the herbicide mixture imazethapyr and imazapic (75 and $25 \mathrm{~g}$ a.i.. ha ${ }^{-1}$ ) and one growing season with non-tolerant rice, without application of imidazolinones; and Succession 2 - three growing seasons using Clearfield system and herbicide application at the same rate specified previously. Factor $B$ included rice cultivars used in the fourth year (BR IRGA 409, IRGA 417, IRGA 422 CL and BRS 7 "TAIM"). Factor C included different herbicides used during the fourth growing season (2006/07), bispyribac-sodium, clomazone + propanyl, cyhalofop-butyl, quinclorac, penoxsulam. Rice production afterusing the Clearfield system requires at least one year without herbicide application (imazethapyr and imazapic) to avoid carryover to non-tolerant rice and yield reduction. A 30\% grain yield reduction is caused by herbicide carryover to non-tolerant rice cultivated in areas where the Clearfield system had been used for three years. No difference was found in the non-tolerant rice cultivars used in this experiment, as all were susceptible to herbicide carryover. No increased injury was observed in rice plants due to herbicide application on the non-tolerant rice cultivars cultivated in rotation. However, it must be emphasized that it is necessary to choose a non-ALS inhibiting herbicide to reduce the pressure of selecting weeds resistant or tolerant to these herbicides.

Keywords: grain yield, plant injury, soil persistence.

\section{INTRODUÇÃO}

O sistema Clearfield ${ }^{\circledR}$ de produção de arroz irrigado, que consiste na utilização de cultivar tolerante aos herbicidas do grupo das imidazolinonas, é uma estratégia eficiente para o controle de arroz-vermelho em lavouras de arroz irrigado (Steele et al., 2002; Ottis et al., 2003; Villa et al., 2006b). No Brasil, o herbicida recomendado para esse sistema é o $\mathrm{Only}^{\circledR}$, composto pela mistura formulada dos herbicidas imazethapyr + imazapic, nas concentrações de 75 e 25 g i.a. $\mathrm{L}^{-1}$, respectivamente (SOSBAI, 2007).

Os herbicidas do grupo químico das imidazolinonas podem persistir na água (Santos et al., 2008) e no solo, tendo sido observado residual no solo por até dois anos (Renner et al., 1998), com potencial de ocasionar fitotoxicidade à cultura semeada em rotação que não seja tolerante aos herbicidas (Ball et al., 2003). Estudos indicam a ocorrência de elevada fitotoxicidade ao arroz em fases iniciais de desenvolvimento (Santos, 2006; Kraemer, 2008), prejudicando o estabelecimento de culturas sucessoras não tolerantes; a intensidade da fitotoxicidade é dependente de fatores de manejo que afetam a degradação do herbicida (Avila et al., 2006).

Apesar da orientação da pesquisa para que o sistema Clearfield seja utilizado por até dois anos consecutivos, seguido de rotação de culturas ou pousio do solo, muitos produtores acabam usando a tecnologia por mais de dois anos ou acabam retornando após dois anos com cultivares de arroz não tolerantes na mesma área de cultivo. Há relatos de persistência do herbicida e dano à cultura do arroz irrigado não tolerante cultivada em sucessão, com niveis de redução de rendimento de grãos que variam de 53 a $72 \%$ (Santos, 2006). Em outros casos, apesar da ocorrência de fitotoxicidade inicial de $28 \%$, o arroz se recuperou e não foi verificada redução na produtividade de seus grãos (Kraemer, 2008).

A fitotoxicidade do residual dos herbicidas sobre arroz não tolerante pode ser alterada pelos herbicidas utilizados no cultivo em sucessão; dependendo de seu modo de ação e da sua seletividade, pode aumentar a fitotoxicidade e comprometer o rendimento de grãos do arroz. Faz-se necessário, assim, gerar informações sobre como migrar desse sistema para o sistema convencional de cultivo de arroz irrigado, com o mínimo de efeito negativo sobre o arroz irrigado não tolerante semeado em sucessão. Em vista do exposto, o objetivo deste experimento foi o de avaliar alternativas de cultivares e herbicidas para o retorno da produção de arroz não tolerante após o uso do sistema Clearfield.

\section{MATERIAL E MÉTODOS}

O experimento foi conduzido em área de várzea sistematizada da Universidade Federal de Santa Maria (Santa Maria-RS) em solo 
classificado como Planossolo Hidromórfico eutrófico arênico, de textura média $\left(\mathrm{pH}_{\text {agua }}(1: 1)\right.$ $=5,0 ; \mathrm{P}=9,3 \mathrm{mg} \mathrm{dm}^{-3} ; \mathrm{K}=72 \mathrm{mg} \mathrm{dm}^{-3} ;$ argila $=23 \% ; \mathrm{MO}=2,1 \% ; \mathrm{Ca}=3,6 \mathrm{cmol}_{\mathrm{c}} \mathrm{dm}^{-3} ; \mathrm{Mg}=$ $0,9 \mathrm{cmol}_{\mathrm{c}} \mathrm{dm}^{-3} ; \mathrm{Al}=0,8 \mathrm{cmol}_{\mathrm{c}} \mathrm{dm}^{-3}$ ).

O delineamento experimental foi de blocos ao acaso, em esquema trifatorial, com três repetições. O fator A foi composto por dois sistemas de sucessão de cultivo de arroz irrigado nos primeiros três anos de cultivo (2003/04, 2004/05 e 2005/06): sistema de sucessão 1 - duas safras agrícolas com o uso do sistema Clearfield e aplicação de imazethapyr + imazapic (75 e 25 g i.a. ha $\left.{ }^{-1}\right)$ e uma safra com cultivo de arroz não tolerante, com aplicaçao de quinclorac; e sistema de sucessão 2 - três safras agrícolas com o uso do sistema Clearfield e aplicação do herbicida na mesma dose especificada anteriormente. $\mathrm{O}$ fator $\mathrm{B}$ constituiu-se de cultivares de arroz irrigado (BR-IRGA 409, IRGA 417, IRGA 422 CL e BRS 7 "TAIM"). O cultivar IRGA $422 \mathrm{CL}$ foi utilizado como testemunha tolerante aos herbicidas do grupo químico das imidazolinonas. $\mathrm{O}$ fator $\mathrm{C}$ foi representado por diferentes herbicidas aplicados na safra 2006/07: bispyribac-sodium ( 48 g i.a. ha ${ }^{-1}$ ); clomazone + propanil $\left(300+1.800\right.$ g i.a. ha $\left.{ }^{-1}\right)$; cyhalofopbutyl (270 g i.a. ha-1); quinclorac (375 g i.a. ha-1); e penoxsulam (48 g i.a. ha-1).

$\mathrm{O}$ arroz foi semeado no sistema convencional, em 21/10/2006, com semeadora de 11 linhas espaçadas de $0,17 \mathrm{~m}$ e $4,5 \mathrm{~m}$ de comprimento, na densidade de $110 \mathrm{~kg} \mathrm{ha}^{-1} \mathrm{de}$ semente. A adubação de base foi de $17,5 \mathrm{~kg}^{-1}$ de nitrogênio (N), $70 \mathrm{~kg} \mathrm{ha}^{-1}$ de $\mathrm{P}_{2} \mathrm{O}_{5}$ e $105 \mathrm{~kg} \mathrm{ha}^{-1}$ de $\mathrm{K}_{2} \mathrm{O}$. A emergência das plantas

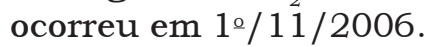

A aplicação dos herbicidas foi realizada aos 16 dias após a emergência (DAE), com um pulverizador costal pressurizado $\operatorname{com} \mathrm{CO}_{2}$ munido de pontas leque 11002 , na vazão de $150 \mathrm{~L} \mathrm{ha}^{-1}$. Um dia após a aplicação dos herbicidas, a área foi inundada, mantendo-se lâmina d'água constante de aproximadamente $5 \mathrm{~cm}$ de altura. $\mathrm{O} \mathrm{N}$ foi aplicado na forma de ureia, dividido em três épocas: a primeira na semeadura, a segunda ( $80 \mathrm{~kg} \mathrm{ha}^{-1}$ de N) um dia antes da inundação e a terceira $\left(40 \mathrm{~kg} \mathrm{ha}^{-1}\right.$ de N) na diferenciação da panícula. Os demais tratos culturais foram conduzidos conforme a recomendação técnica para a cultura (SOSBAI, 2007).

Aos $14 \mathrm{DAE}$, determinou-se a densidade inicial de plantas através da contagem da sua população em um metro de comprimento da linha de semeadura. Nessa mesma área, foram determinados a estatura de plantas e o número de panículas por planta aos $30 \mathrm{DAE} e$ no momento da colheita. A avaliação de fitotoxicidade no arroz foi realizada aos 15, 21 e $45 \mathrm{DAE}$, utilizando uma escala de 0 a $100 \%$, em que $0=$ sem fitotoxicidade e $100=$ morte das plantas. A produtividade de grãos foi estimada através da colheita manual, em área de $6,88 \mathrm{~m}^{2}(4,5 \times 1,53 \mathrm{~m})$, quando os grãos apresentavam umidade média de $22 \%$. Após a trilha, limpeza e pesagem dos grãos com casca, os dados foram corrigidos para $13 \%$ de umidade e convertidos em $\mathrm{kg} \mathrm{ha}^{-1}$.

Os dados foram submetidos aos testes das pressuposições do modelo matemático, e em seguida foram submetidos à análise de variância (ANOVA, $\mathrm{P} \leq 0,05$ ). Após as médias foram comparadas pelo teste de Tukey $(P \leq 0,05)$. Antes da análise, os dados de fitotoxicidade foram transformados em $\mathrm{yt}=\operatorname{arcoseno} \sqrt{\frac{y+0.5}{100}}$.

\section{RESULTADOS E DISCUSSÃO}

O estande inicial de plantas (Tabela 1) foi similar entre os cultivares e não foi afetado pelos manejos da área e pelos herbicidas aplicados na safra 2006/07, com média de 177 plantas $\mathrm{m}^{-2}$. A primeira avaliação de fitotoxicidade foi realizada antes do início da irrigação e da aplicação dos herbicidas, com sintomas visuais de fitotoxicidade aparente apenas na área com três anos de aplicação do herbicida composto pela mistura formulada de imazethapyr + imazapic (sistema de sucessão 2). Foi observada fitotoxicidade em todos os cultivares, inclusive no tolerante, porém com valores maiores nos não tolerantes (BR-IRGA 409, IRGA 417 e BRS 7 "TAIM"). Segundo Ball et al. (2003), pode haver fitotoxicidade decorrente do residual do herbicida no solo, dependendo da cultura sucessora. A fitotoxicidade inicial observada no cultivar tolerante IRGA $422 \mathrm{CL}$ se deve ao fato de que 
a tolerância desse cultivar às imidazolinonas é dependente da dose do herbicida e dos fatores climáticos. A fitotoxicidade inicial dos herbicidas do grupo das imidazolinonas sobre cultivares de arroz tolerante tem sido demonstrada por vários autores (Ottis et al., 2003; Villa et al., 2006a).

Após o início da irrigação do arroz (21 DAE), não foi observada diferença de fitotoxicidade entre os cultivares no sistema de sucessão 1 . Contudo, no sistema de sucessão 2, todos os cultivares apresentaram aumento nos sintomas de fitotoxicidade após a entrada de água na área, mesmo no cultivar tolerante IRGA $422 \mathrm{CL}$, que apresentou o menor valor de fitotoxicidade entre os cultivares avaliados. Com relação aos herbicidas aplicados em 2006/07, a fitotoxicidade foi maior nas plantas submetidas ao tratamento com clomazone + propanil e no tratamento com bispyribacsodium, independentemente do sistema de sucessão empregado. O aumento da fitotoxicidade observado após a entrada de água na área pode ser decorrente do aumento da disponibilidade dos herbicidas imazethapyr + imazapic com o incremento do teor de água no solo (Avila et al., 2006).

$\mathrm{Na}$ avaliação aos $40 \mathrm{DAE}$, houve redução na fitotoxicidade dos cultivares nos dois sistemas de sucessão, não havendo diferenças de fitotoxicidade entre os cultivares na área com o sistema de sucessão 1 . No entanto, na área com o sistema de sucessão 2 os cultivares não tolerantes apresentaram maior fitotoxicidade quando comparados ao tolerante IRGA $422 \mathrm{CL}$. Analisando os herbicidas aplicados, observase diferença de fitotoxicidade entre os tratamentos; o herbicida cyhalofop-butyl causou menor fitotoxicidade quando comparado com os demais.

Comparando os dois sistemas de sucessão adotados nas áreas, o sistema 2, com três anos consecutivos de aplicação do herbicida composto pela mistura formulada de imazethapyr + imazapic, sempre apresentou maior fitotoxicidade que o sistema de sucessão 1 , que teve

Tabela 1 - Estande inicial de plantas e toxicidade de cinco herbicidas em quatro cultivares de arroz irrigado semeados em 2006/07 após o uso do sistema Clearfield $\mathbb{R}$, em área com dois sistemas de sucessão de cultivo de arroz irrigado e de herbicidas. Santa Maria-RS. 2008

\begin{tabular}{|c|c|c|c|c|c|c|c|c|}
\hline \multirow{3}{*}{ Cultivar } & \multirow{2}{*}{\multicolumn{2}{|c|}{$\begin{array}{c}\text { Estande inicial } \\
\left(\text { plantas } \mathrm{m}^{-2}\right)\end{array}$}} & \multicolumn{6}{|c|}{ Fitotoxicidade (\%) } \\
\hline & & & \multicolumn{2}{|c|}{$15 \mathrm{DAE}^{1 /}$} & \multicolumn{2}{|c|}{$21 \mathrm{DAE}$} & \multicolumn{2}{|c|}{$40 \mathrm{DAE}$} \\
\hline & Sucessão $1^{2 /}$ & Sucessão $2^{3 /}$ & Sucessão $1^{2 !}$ & Sucessão $2^{3 /}$ & Sucessão $1^{2 /}$ & Sucessão $2^{3 /}$ & Sucessão $1^{2 /}$ & Sucessão $2^{\frac{3}{3}}$ \\
\hline BR-IRGA 409 & $177^{\mathrm{ns}}$ & $153^{\mathrm{ns}}$ & 0 & $57 \mathrm{a}$ & $20^{\mathrm{ns}}$ & $74 \mathrm{a}$ & $11^{\mathrm{ns}}$ & $69 \mathrm{a}$ \\
\hline IRGA 417 & 166 & 153 & 0 & $55 \mathrm{a}$ & 26 & $80 \mathrm{a}$ & 19 & $73 \mathrm{a}$ \\
\hline IRGA 422 CL & 217 & 204 & 0 & $7 \mathrm{~b}$ & 13 & $17 \mathrm{~b}$ & 6 & $8 \mathrm{~b}$ \\
\hline BRS 7 "TAIM" & 182 & 172 & 0 & $48 \mathrm{a}$ & 23 & $81 \mathrm{a}$ & 16 & $71 \mathrm{a}$ \\
\hline \multicolumn{9}{|l|}{ Tratamentos ${ }^{4}$} \\
\hline Testemunha & $202^{\mathrm{ns}}$ & $175^{\text {ns }}$ & $---\frac{5}{\frac{5}{1}}$ & -- & $0 \mathrm{~d}$ & $55 \mathrm{c}$ & $0 \mathrm{c}$ & $48 \mathrm{c}$ \\
\hline Bispyribac-sodium & 172 & 172 & --- & --- & $28 \mathrm{ab}$ & $67 \mathrm{ab}$ & $19 \mathrm{a}$ & $60 \mathrm{a}$ \\
\hline Clomazone + propanil & 198 & 176 & --- & --- & $33 \mathrm{a}$ & $72 \mathrm{a}$ & $18 \mathrm{a}$ & $60 \mathrm{a}$ \\
\hline Cyhalofop-butyl & 178 & 163 & --- & --- & $15 \mathrm{~cd}$ & $59 \mathrm{bc}$ & $8 \mathrm{~b}$ & $53 \mathrm{~b}$ \\
\hline Quinclorac & 170 & 181 & --- & --- & $23 \mathrm{c}$ & $64 \mathrm{bc}$ & $18 \mathrm{a}$ & $56 \mathrm{a}$ \\
\hline Penoxsulam & 189 & 155 & --- & --- & $25 \mathrm{~b}$ & $64 \mathrm{bc}$ & $18 \mathrm{a}$ & $59 \mathrm{a}$ \\
\hline Média & A 184 & A 170 & B 0 & A 41 & B 21 & A 63 & B 13 & A 55 \\
\hline $\mathrm{CV} \%$ & \multicolumn{2}{|c|}{12,9} & \multicolumn{2}{|c|}{12,3} & \multicolumn{2}{|c|}{13,3} & \multicolumn{2}{|c|}{17,7} \\
\hline
\end{tabular}

1/ Dias após a emergência das plantas; ${ }^{2 /}$ Sistema de sucessão de cultivo de arroz irrigado, com cultivo de arroz tolerante e aplicação de $1 \mathrm{~L} \mathrm{ha}^{1}$ do herbicida composto pela mistura formulada de imazethapyr e imazapic $\left(75+25 \mathrm{~g}\right.$ i.a. ha $\left.{ }^{-1}\right)$, em dois anos agrícolas $(2003 / 04 \mathrm{e}$ 2004/05), e cultivo de arroz não tolerante e aplicação do herbicida quinclorac no último ano (2005/06) antes da instalação do experimento; ${ }^{3 /}$ Sistema de sucessão de cultivo de arroz irrigado, com cultivo de arroz tolerante e aplicação de $1 \mathrm{~L}$ ha ${ }^{1}$ do herbicida composto pela mistura formulada de imazethapyr e imazapic $\left(75+25\right.$ g i.a L $\left.{ }^{-1}\right)$, nos três anos agrícolas que antecederam o experimento $(2003 / 04,2004 / 05$ e 2005/06); ${ }^{4 /}$ Aplicação em pós-emergência com o arroz no estádio $\mathrm{V}_{5}$ (Counce et al., 2000); ${ }^{4 /}$ Bispyribac-sodium na dose de $48 \mathrm{~g}$ i.a. ha ${ }^{-1}$; Clomazone na dose de $300 \mathrm{~g}_{\text {i.a. }} \mathrm{ha}^{-1}$ + Propanil na dose de $1.800 \mathrm{~g}^{\text {i.a. }} \mathrm{ha}^{-1}$; Cyhalofop-butyl na dose de $270 \mathrm{~g}$ i.a. ha ${ }^{-1}$; Quinclorac na dose de 375 g i.a. ha ${ }^{-1}$; e Penoxsulam na dose de 48 g i.a. ha ${ }^{-1} ;{ }^{5}$ Dados não avaliados pois os tratamentos ainda não haviam sido aplicados; ${ }^{\text {ns }}$ Teste F não significativo a $5 \%$ de probabilidade de erro; * Médias seguidas por distintas letras, minúscula na coluna e maiúscula na linha, diferem entre si pelo teste de Tukey a $5 \%$ de probabilidade. 
apenas dois anos com aplicação do herbicida e um com aplicação de outro herbicida sem efeito residual. Esse resultado demonstra que a fitotoxicidade do residual dos herbicidas imazethapyr + imazapic em arroz irrigado não tolerante cultivado em sucessão pode ser reduzida com apenas um ano sem a aplicação do herbicida.

A estatura das plantas dos cultivares de arroz irrigado avaliada aos $30 \mathrm{DAE}$ (Tabela 2) teve comportamento diferenciado nos sistemas de sucessão. No sistema de sucessão 1 não houve diferença entre os cultivares estudados. Contudo, no sistema 2, onde houve aplicação do herbicida composto pela mistura formulada de imazethapyr + imazapic por três anos, os cultivares suscetiveis tiveram menor estatura que o tolerante ao herbicida (IRGA $422 \mathrm{CL}$ ), devido ao efeito do residual das imidazolinonas nas plantas. Quando se comparam os dois manejos, a estatura de plantas foi superior no sistema de sucessão $1 \mathrm{em}$ relação ao 2, porém, na avaliação antecedendo a colheita, não foram observadas diferenças entre herbicidas, sistemas ou cultivares.

O número de panículas por metro quadrado foi similar entre os cultivares semeados no sistema de sucessão 1. Entretanto, houve diferença entre os cultivares no sistema de sucessão 2, onde o cultivar IRGA 422 CL apresentou maior número de panículas por metro quadrado. Comparando sistemas de sucessão, foi observado menor número de panículas por metro quadrado na área onde foi aplicado, por três anos consecutivos, $1 \mathrm{~L} \mathrm{ha}^{-1}$ do herbicida composto pela mistura formulada de imazethapyr + imazapic (75 e 25 g i.a. ha $\left.{ }^{-1}\right)$ (sucessão 2). Isso ocorre, provavelmente, pela redução do número de perfilhos por área, causada pela morte precoce de plantas, após a entrada de água e pelo menor perfilhamento das plantas devido à fitotoxicidade do residual dos herbicidas. Esses resultados corroboram os de Villa et al. (2006a).

Tabela 2 - Estatura de plantas, número de panículas por metro quadrado e produtividade de grãos de quatro cultivares de arroz irrigado semeados em 2006/07 após o uso do sistema Clearfield ${ }^{\circledR}$ em área com dois sistemas de sucessão de cultivo de arroz irrigado e de herbicidas. Santa Maria-RS. 2008

\begin{tabular}{|c|c|c|c|c|c|c|c|c|}
\hline \multirow{3}{*}{ Cultivar } & \multicolumn{4}{|c|}{ Estatura de plantas $(\mathrm{cm})$} & \multirow{2}{*}{\multicolumn{2}{|c|}{$\frac{\text { Panícula }}{\left(\text { número } \mathrm{m}^{-2}\right)}$}} & \multirow{2}{*}{\multicolumn{2}{|c|}{$\begin{array}{c}\text { Produtividade de grãos } \\
\left(\mathrm{kg} \mathrm{ha}^{-1}\right)\end{array}$}} \\
\hline & \multicolumn{2}{|c|}{$30 \mathrm{DAE}^{1 / /}$} & \multicolumn{2}{|c|}{ Antecedendo a colheita } & & & & \\
\hline & Sucessão $1^{2 / 1}$ & Sucessão $2^{\frac{3}{}}$ & Sucessão $1^{\underline{2}}$ & Sucessão $2^{\frac{3}{}}$ & Sucessão $1^{2 /}$ & Sucessão $2^{\frac{3}{}}$ & Sucessão $1^{2 / /}$ & Sucessão $2^{\frac{3}{}}$ \\
\hline BR-IRGA 409 & $22^{\mathrm{ns}}$ & $13 \mathrm{~b}$ & $92^{\mathrm{ns}}$ & $86^{\mathrm{ns}}$ & $457^{\mathrm{ns}}$ & $342^{b}$ & A $8.795^{\text {ns }}$ & B $5.121 \mathrm{~b}$ \\
\hline IRGA 417 & 23 & $12 \mathrm{~b}$ & 90 & 85 & 464 & $313^{b}$ & A 8.620 & B $4.989 \mathrm{~b}$ \\
\hline IRGA $422 \mathrm{CL}$ & 25 & $27 \mathrm{a}$ & 88 & 84 & 468 & $440^{\mathrm{a}}$ & A 8.632 & A $7.277 \mathrm{a}$ \\
\hline BRS 7 "TAIM" & 20 & $12 \mathrm{~b}$ & 87 & 83 & 452 & $301^{b}$ & A 8.609 & B 5.088 b \\
\hline \multicolumn{9}{|l|}{ Tratamentos ${ }^{4}$} \\
\hline Testemunha & $24^{\mathrm{ns}}$ & $15^{\mathrm{ns}}$ & $85^{\text {ns }}$ & $85^{\text {ns }}$ & $431^{\text {ns }}$ & $308^{\text {ns }}$ & $8.899^{\text {ns }}$ & $5.959^{\text {ns }}$ \\
\hline Bispyribac-sodium & 21 & 16 & 87 & 88 & 484 & 321 & 8.655 & 5.981 \\
\hline Clomazone + propanil & 22 & 15 & 87 & 85 & 424 & 308 & 8.806 & 5.728 \\
\hline Cyhalofop-butyl & 22 & 17 & 88 & 85 & 412 & 333 & 8.518 & 5.016 \\
\hline Quinclorac & 25 & 16 & 89 & 86 & 450 & 326 & 8.564 & 5.252 \\
\hline Penoxsulam & 22 & 16 & 86 & 83 & 482 & 333 & 8.524 & 5.776 \\
\hline Média & A 23 & B 16 & A 88 & A 85 & A 453 & B 318 & A 8.663 & B 5.619 \\
\hline $\mathrm{CV} \%$ & \multicolumn{2}{|c|}{13,6} & \multicolumn{2}{|c|}{4,5} & \multicolumn{2}{|c|}{14,2} & \multicolumn{2}{|c|}{15,7} \\
\hline
\end{tabular}

${ }^{1 /}$ Dias após a emergência das plantas; ${ }^{2 /}$ Sistema de sucessão de cultivo de arroz irrigado, com cultivo de arroz tolerante com a aplicação de $1 \mathrm{~L} \mathrm{ha}{ }^{1}$ do herbicida composto pela mistura formulada de imazethapyr e imazapic $\left(75+25 \mathrm{~g}\right.$ i.a. $\left.\mathrm{L}^{-1}\right)$, em dois anos agrícolas $(2003 / 04 \mathrm{e}$ 2004/05), e cultivo de arroz não tolerante e aplicação do herbicida quinclorac no último ano (2005/06) antes da instalação do experimento; ${ }^{3 /}$ Sistema de sucessão de cultivo de arroz irrigado, com cultivo de arroz tolerante e aplicação de $1 \mathrm{~L}$ ha ${ }^{1}$ do herbicida composto pela mistura formulada de imazethapyr e imazapic $\left(75+25 \mathrm{~g}\right.$ i.a L $\left.{ }^{-1}\right)$, nos três anos agrícolas que antecederam o experimento $(2003 / 04$, 2004/05 e 2005/06); " $/$ Aplicação em pós-emergência com o arroz no estádio $\mathrm{V}_{5}$ (Counce et al., 2000); ${ }^{4 /}$ Bispyribac-sodium na dose de $48 \mathrm{~g}$ i.a. ha ${ }^{-1}$; Clomazone na dose de 300 g i.a. ha ${ }^{-1}+$ Propanil na dose de 1.800 g i.a. ha ${ }^{-1}$; Cyhalofop-butyl na dose de 270 g i.a. ha ${ }^{-1}$; Quinclorac na dose de 375 g i.a. ha ${ }^{-1}$; e Penoxsulam na dose de 48 g i.a. ha ${ }^{-1}$; ${ }^{\text {ns Teste }} \mathrm{F}$ não significativo a $5 \%$ de probabilidade; * Médias seguidas por distintas letras, minúscula na coluna e maiúscula na linha, diferem entre si pelo teste de Tukey a $5 \%$ de probabilidade. 
Os cultivares de arroz irrigado apresentaram produtividade de grãos de forma variável com o sistema de sucessão empregado. No sistema de sucessão 1 , não houve diferença de rendimento entre os cultivares; contudo, no sistema de sucessão 2, que recebeu três anos consecutivos do herbicida composto da mistura formulada de imazethapyr + imazapic, houve menor produtividade dos cultivares não tolerantes quando comparados com o IRGA $422 \mathrm{CL}$. A redução média do rendimento de grãos dos cultivares não tolerantes foi de $30 \%$ em relação à do cultivar tolerante IRGA 422 CL. Essa redução do rendimento de grãos se deve ao residual dos herbicidas imazethapyr + imazapic, pois o cultivar IRGA $422 \mathrm{CL}$ é tolerante aos herbicidas. Isso ocorre porque o residual desses herbicidas altera o desenvolvimento das plantas, causando-lhes a morte mesmo em áreas cultivadas após um ano de uso do sistema Clearfield (Villa et al., 2006a). Dados similares foram encontrados por Zhang et al. (2002), que verificaram reduções de até $41 \%$ na produtividade de grãos do arroz não tolerante semeado após o uso de imazethapyr.

A produtividade de grãos não foi alterada pela aplicação dos herbicidas na safra 2006/07 e não houve diferença entre estes em ambos os manejos empregados, demonstrando que o aumento da fitotoxicidade inicial causada pela aplicação de determinados herbicidas não foi o suficiente para causar redução de rendimento de grãos, demonstrando assim a habilidade das plantas de arroz irrigado em superar essas fitotoxicidades.

Analisando os cultivares não tolerantes entre os dois sistemas de sucessão, a área do sistema de sucessão 1 apresentou produtividade média $42 \%$ superior (8.675 $\left.\mathrm{kg} \mathrm{ha}^{-1}\right)$ à obtida pelos mesmos cultivares na área com três anos de aplicação do herbicida (sistema de sucessão $2=5.066 \mathrm{~kg} \mathrm{ha}{ }^{1}$ ), indicando menor efeito residual após um ano sem o uso do herbicida composto pela mistura formulada de imazethapyr + imazapic. Deve-se ressaltar que, segundo alguns pesquisadores (Renner et al., 1998; Donald, 2006), as imidazolinonas podem apresentar residual no solo por até dois anos em solos de várzeas.

A persistência de herbicidas do grupo das imidazolinonas por mais de um ano após a aplicação já havia sido relatada por autores como Moyer \& Esau (1996), os quais observaram que imazethapyr causa diminuição de produtividade em canola até dois anos após a aplicação e três anos para batata e beterraba. Cabe destacar que o efeito residual dos herbicidas do grupo das imidazolinonas pode apresentar diferentes prejuízos a culturas não tolerantes, variando conforme o manejo da área, o clima, a umidade do solo, a quantidade de herbicida aplicado, entre outros fatores que contribuem para a sua persistência no solo. Os danos registrados para o efeito do residual desses herbicidas vão desde redução no estande da planta e da massa seca (Renner et al., 1988; Zhang et al., 2002; Matocha et al., 2003; Villa et al., 2006a), do tamanho das raízes (Wixson \& Shaw, 1992), até redução da produtividade, como demonstrado por esta pesquisa e por outros autores (Loux $\&$ Reese, 1993; Zhang et al., 2002).

Conclui-se que os sistemas de sucessão de arroz com dois anos de aplicação do herbicida composto pela mistura formulada de imazethapyr + imazapic requerem, pelo menos, uma safra agrícola sem o uso do herbicida, para evitar que o seu residual altere a produtividade de grãos de cultivares não tolerantes.

A redução de produtividade do arroz não tolerante causada pelo residual do herbicida (imazethapyr + imazapic) semeado após três anos de uso do sistema Clearfield foi de, em média, $30 \%$.

Quando se retornou ao sistema de cultivo com cultivares convencionais, não houve diferença de produtividade de grãos entre os cultivares não tolerantes testados neste experimento, pois todos se mostraram suscetiveis ao residual dos herbicidas imazethapyr + imazapic.

No que se refere ao cultivo de arroz não tolerante, não houve aumento da fitotoxicidade em plantas de arroz pelo uso dos herbicidas bispyribac-sodium, clomazone + propanil, cyhalofop-butyl, quinclorac e penoxsulam a ponto de alterar o rendimento de grãos. Contudo, na escolha do herbicida deve-se levar em conta a rotação de modos de ação, evitando-se assim o uso de produtos cujo modo de ação seja a inibição da enzima ALS logo após o uso do sistema Clearfield. 


\section{AGRADECIMENTO}

Ao CNPq, pela concessão de bolsa de iniciação científica, e de produtividade em pesquisa dos Profs. Luís Avila e Enio Marchesan. À UFSM, e ao FONTAGRO, pelo auxilio financeiro.

\section{LITERATURA CITADA}

AVILA, L. et al. Imazethapyr aqueous photolysis, reaction quantum yield, and hydroxyl radical rate constant. J. Agric. Food. Chem., v. 54, n. 7, p. 2635-2639, 2006

BALL, D. A. et al. Effect of imazamox soil persistence on dryland rotational crops. Weed Technol., v. 17, n. 1, p. 161-165, 2003.

COUNCE, P. A.; KEISLING, T. C.; MITCHELL, A. J. A uniform, objective, and adaptive system for expressing rice development. Crop Sci., v. 40, n. 2, p. 436-443, 2000.

DONALD, W. Estimated corn yields using either weed cover or rated control after pre-emergence herbicides. Weed Sci., v. 54, n. 2 , p. $373-379,2006$

LOUX, M. M.; REESE, K. D. Effect of soil type and pH on persistence and carryover of imidazolinone herbicides. Weed Technol., v. 7, n. 2, p. 452-458,1993.

KRAEMER, A.F. Residual da mistura formulada dos herbicidas imazethapyr e imazapic em áreas de arroz sob diferentes manejos de solo. 2008. 65 f. Dissertação (Mestrado em Agronomia) - Universidade Federal de Santa Maria, Santa Maria, 2008.

MATOCHA, M. A. et al. The persistence of imazapic in peanut (Arachis hypogaea) crop rotations. Weed Technol., v. 17, n. 2 , p. $325-329,2003$

MOYER, J. R.; ESAU, R. Imidazolinone herbicide effects on following rotational crops in Southern Alberta. Weed Technol., v. 10, n. 1, p. 100-106, 1996.
OTTIS, B. V. et al. Imazethapyr application methods and sequences for imidazolinone-tolerant rice (Oryza sativa). Weed Technol., v. 17, n. 3, p. 526-533, 2003.

RENNER, K. A. et al. Effect of tillage application method on corn (Zea mays) response to imidazolinone residues in soil. Weed Technol., v. 12, n. 2, p. 281-285, 1998.

SANTOS, F. M. Alternativas de controle químico do arroz-vermelho e persistência dos herbicidas (imazethapyr + imazapic) e clomazone na água e no solo 2006. 73 f. Dissertação (Mestrado em Agronomia) -

Universidade Federal de Santa Maria, Santa Maria, 2006

SANTOS, F. M. et al. Persistência dos herbicidas imazethapyr e clomazone em lâmina de água do arroz irrigado Planta Daninha, v. 26, n. 4, p. 875-881, 2008.

SOCIEDADE SUL-BRASILEIRA DE ARROZ IRRIGADO - SOSBAI. Arroz irrigado: recomendações técnicas da pesquisa para o Sul do Brasil. Pelotas: 2007.154 p.

STEELE, G.L. et al. Control of red rice (Oryza sativa) in imidazolinone-tolerant rice (O. sativa). Weed Technol., v. 16, n. 3, p. 627-630, 2002.

VILLA, S. C. C. et al. Controle de arroz-vermelho em dois genótipos de arroz (Oryza sativa) tolerante/resistente a herbicidas do grupo das imidazolinonas. Planta Daninha, v. 24, n. 3 , p. $549-555,2006$ a.

VLLLA, S. C. C. et al. Arroz tolerante a imidazolinonas: controle do arroz-vermelho, fluxo gênico e efeito residual do herbicida em culturas sucessoras não tolerantes.

Planta Daninha, v. 24, n. 4, p. 761-768, 2006 b.

WIXSON, M. B.; SHAW, D. R. Effect of soil-applied AC 263222 on crop rotated with soybean. Weed Technol., v. 6, n. 2, p. 276-279, 1992.

ZHANG, W. et al. Rice (Oryza sativa) response to rotational crop and rice herbicide combinations. Weed Technol., v. 16, n. 2, p. 340-345, 2002. 